References:

[1] Özyürek, S., D. Bayraktar, and A. Genç, Are the alterations in body posture related to decreased trunk muscle endurance in healthy young adults? Journal of back and musculoskeletal rehabilitation, 2018. 31(3): p. 431-436.

[2] McGill, S.M., Low back stability: from formal description to issues for performance and rehabilitation. Exercise and sport sciences reviews, 2001. 29(1): p. 26-31.

Disclosure of Interests: None declared

DOI: 10.1136/annrheumdis-2020-eular.5685

\section{THU0634-HPR KNEE EXTENSOR MUSCLE STEADINESS IN RELATION TO MAXIMAL TORQUE AND PHYSICAL FUNCTIONING IN PATIENTS WITH KNEE OSTEOARTHRITIS}

A. Satam ${ }^{1}$, M. Van der Leeden2, A. De Zwart2, S. Verberne2, J. Schrijvers ${ }^{3}$, J. Dekker ${ }^{4}$, W. Lems ${ }^{5}$, J. Haarlaar ${ }^{3}$, M. Van der Esch6. ${ }^{1}$ Reade, Amsterdam, Netherlands; ${ }^{1}$ Reade, Amsterdam, Netherlands; ${ }^{3}$ Amsterdam UMC, VU Medical Centre, Rehabilitation, Amsterdam, Netherlands; ${ }^{4}$ Amsterdam UMC VU Medical Centre, Rehabilitation, Amsterdam, Netherlands; ${ }^{5}$ Amsterdam UMC VU Medical Centre, Rheumatology, Amsterdam, Netherlands; ${ }^{1}$ Reade, Amsterdam, Netherlands

Background: Osteoarthritis (OA) of the knee is characterized by knee pain and limitations in daily activities. Muscle weakness is associated with these characteristics, quantified as maximal voluntary muscle torque (MVT). The quality of muscle contraction is presented by fluctuations observed on a torque-time curve and the extent of these fluctuations is referred to as muscle steadiness. Whether muscle steadiness is associated with maximal muscle torque and consequently with pain and activity limitations is unknown.

Objectives: To determine the association of knee extensor muscle steadiness with MVT and to explore the association of muscle steadiness with physical functioning in subjects with knee OA.

Methods: Baseline data of 172 patients out of 177 patients with knee OA, who participated in the VIDEX trial (trial registration number, NL47786.048.14), were used for this study. Maximal voluntary knee extension torque (MVT) was assessed using an isokinetic dynamometer. Torque-time curve data were processed into (i) coefficient of magnitude of torque variance (CV) in percentage (\%), (ii) frequency of torque variance as peak power frequency (PPF) in Hertz $(\mathrm{Hz})$ and (iii) MVT in Newton meters (Nm). Physical functioning was assessed using the Western Ontario and McMaster Universities Osteoarthritis Index (WOMAC) questionnaire, the Get-Up \& Go (GUG) test, the 6-minute walk test (6MWT) and the Stair climb up \& down test. Correlation and Regression analyses were performed to determine associations. Sex, age, BMI, KL-grade, knee alignment and pain were considered as potential confounders.

Results: Lower CV and PPF, reflecting better muscle steadiness, were significantly associated ( $p<0.01$ and $p<0.05$, respectively) with higher MVT, but associations were weak. Regression analyses showed a significant association of lower $C V$ with better physical functioning on the WOMAC $(p<0.05)$, also after correction for relevant confounders. The association with WOMAC was confounded by pain, but not by sex, age and BMI. No associations of CV with the GUG test, the 6MWT and the Stair climb up \& down test were found. PPF was not significantly associated with physical functioning.

Conclusion: This is the first explorative study of muscle steadiness in relation to physical functioning in knee OA patients. Muscle steadiness is, to some extent, related to better physical functioning, but this is not consistent across all measures of physical functioning in this study. There seems to be some relationship, but it is weak and needs further exploration. No previous studies comparing clinical scores to muscle steadiness in knee OA were found to compare our results. Studies on muscle steadiness are needed to improve our understanding on this aspect of muscle torque.

References: N/A

Disclosure of Interests: Anuja Satam: None declared, Marike van der Leeden: None declared, Arjan de Zwart: None declared, Simon Verberne: None declared, Jim Schrijvers: None declared, Joost Dekker: None declared, WIllem Lems Grant/research support from: Pfizer, Consultant of: Lilly, Pfizer, Jaap Haarlaar: None declared, Martin van der Esch: None declared

DOI: 10.1136/annrheumdis-2020-eular.3690

\section{THU0635-HPR PREVALENCE AND CLINICAL CHARACTERISTICS OF NEOPLASIA AMONG A COHORT OF PATIENTS WITH SYSTEMIC SCLEROSIS}

J. L. Tandaipan Jaime ${ }^{1}$, E. Riera Alonso ${ }^{1}$, N. Gimenez Gomez ${ }^{2}$

G. Ghio ${ }^{1}$, L. Berbel Arcobe ${ }^{1}$, S. Martinez Pardo ${ }^{1} .{ }^{1}$ Hospital Universitari Mutua Terrassa, Rheumatology, Terrassa, Spain; ${ }^{2}$ Hospital Universitari Mutua Terrassa, Research Support Department, Terrassa, Spain

Background: Patients with Systemic Sclerosis (SSc) have increased risk of malignancy compared to general population. The specific risk factors and underlying physiopathological mechanisms are still unknown, although some studies suggest that a relationship between malignancies and certain antibodies can exist. Lung, breast and hematological cancers are the most frequently seen among these patients.

Objectives: To describe the prevalence of malignancies in a cohort of SSc patients and analyze the epidemiological, clinical and immunological characteristics

Methods: A retrospective observational study was conducted at a tertiary-level university hospital, including a cohort of patients with SSc (ACR/EURLAR 2013 criteria). The main variable was neoplasia prevalence and also, malignancy type, age, evolution of the SSc at the time of diagnosis and mortality were collected. Regarding SSc, demographic data, clinical and immunological characteristics, organ involvement, capillaroscopy findings and presence of other autoimmune diseases were collected.

Results: A $15 \%$ of the 98 patients with SSc presented malignancies $(80 \%$ women). The mean age at the time of diagnosis was $57 \pm 15$ years old (table 1 ) The frequency of cancer was: $40 \%$ breast, $13 \%$ colon, $7 \%$ ovary and lung. 2 patients died (1 breast, 1 lung). The limited subtype (ISSc) was the most frequent $(80 \%)$ and $33 \%$ showed overlap syndrome (26\% Sjögren syndrome) Regarding clinical manifestations: $67 \%$ had telangiectasia, $33 \%$ pitting scars, joint and digestive involvement. Most frequently seen antibodies were: $67 \%$ ant centromere (ACA) and $20 \%$ anti topoisomerase (ATA). None of the patients presented anti-ARN polimerase III (ARN-pol), and 13\% had none of them (triple negative). Active and early capillaroscopy patterns were seen in a $46 \%$ and $27 \%$. $\mathrm{SSc}$ and cancer were diagnosed in less than 5 years difference among a $33 \%$ of the cohort. A relationship between age and cancer was detected $(p=0,042)$ Patients with neoplasia were a mean of 10 years older than those without malignancies (IC95\%: 1-19 years)

Table 1.

\begin{tabular}{|c|c|c|}
\hline & $\begin{array}{l}\text { SSc with neoplasia } \\
\qquad n=15(\%)\end{array}$ & $\begin{array}{l}\text { SSc without neoplasia } \\
\qquad n=83(\%)\end{array}$ \\
\hline Female & $12(80)$ & $76(92)$ \\
\hline Mean age ${ }^{\star}(\mathrm{n} ; \mathrm{DE})$ & $57(15)$ & $52(17)$ \\
\hline Pre-scleroderma & $1(7)$ & $11(13)$ \\
\hline Limited & $12(80)$ & $54(65)$ \\
\hline Diffuse & $2(13)$ & $12(15)$ \\
\hline SINE & 0 & $6(7)$ \\
\hline Overlap syndrome & $5(33)$ & $14(17)$ \\
\hline Sjögren & $4(27)$ & $10(12)$ \\
\hline MCTD & $1(7)$ & $2(2)$ \\
\hline Rheumatoid Arthritis & $1(7)$ & $3(4)$ \\
\hline Myositis & $1(7)$ & 0 \\
\hline \multicolumn{3}{|l|}{ Clinical manifestations } \\
\hline Telangiectasia & $10(67)$ & $41(49)$ \\
\hline Pitting Scars & $5(33)$ & $11(13)$ \\
\hline Joint & $5(33)$ & $27(33)$ \\
\hline Digestive & $5(33)$ & $33(40)$ \\
\hline Digital ulcers & $4(27)$ & $11(13)$ \\
\hline Calcinosis & $4(27)$ & $12(14)$ \\
\hline ILD & $3(20)$ & $16(19)$ \\
\hline $\mathrm{PAH}$ & $3(20)$ & $8(10)$ \\
\hline Cardiac & $3(20)$ & $4(5)$ \\
\hline Muscular & $2(13)$ & $3(4)$ \\
\hline Puffy Fingers & $2(13)$ & $24(29)$ \\
\hline Renal & 0 & $2(2)$ \\
\hline \multicolumn{3}{|l|}{ Antibodies } \\
\hline ACA & $10(67)$ & $46(55)$ \\
\hline ATA & $3(20)$ & $12(14)$ \\
\hline Anti-ARN & 0 & $4(5)$ \\
\hline Triple negative & $2(13)$ & $23(28)$ \\
\hline \multicolumn{3}{|l|}{ Capillaroscopy } \\
\hline Early & $4(27)$ & $19(23)$ \\
\hline Active & $7(46)$ & $39(47)$ \\
\hline Late & 0 & $3(4)$ \\
\hline \multicolumn{3}{|l|}{ Treatment } \\
\hline Calcium antagonists & $11(73)$ & $52(63)$ \\
\hline PPIs & $7(46)$ & $33(40)$ \\
\hline Corticosteroids & $8(53)$ & $24(29)$ \\
\hline DMARD & $5(33)$ & $26(31)$ \\
\hline
\end{tabular}

${ }^{*} \mathrm{P}<0,05$ test $\mathrm{t}$-student

MCTD (Mixed Connective Tissue Disease), ILD (Interstitial Lung Disease), PAH (Pulmonary Artery Hypertension), Triple negative (anti ARN, ACA and ATA negative antibodies), PP (Proton Pump Inhibitor), ACE inhibitors (Angiotensin Converting Enzyme inhibitors), ARBs (Angiotensin II Receptor Blockers), DMARD (Disease-Modifying Anti-Rheumatic Drugs).

Conclusion: Our study showed a similar prevalence of the most frequent neoplasia among patients with SSc compared to general population (around 15\%). This prevalence is similar to other series. The only epidemiological factor related to neoplasia was the age; a major proportion of ISSc was detected but without statistical significance. In a third of the patients there were less than 5 years 
of difference between cancer and SSc diagnosis. No association was found between neoplasia and certain antibodies. We recommend further studies to evaluate the relationship between SSc and cancer.

Disclosure of Interests: None declared

DOI: 10.1136/annrheumdis-2020-eular.4827

\section{THU0636-HPR REMS TECHNOLOGY APPLIED TO RHEUMATIC DISEASE}

M. D. Tomai Pitinca ${ }^{1}$, C. Caffarelli ${ }^{2}$, S. Gonnelli ${ }^{2}{ }^{1}$ National Research Council, Institute of Clinical Physiology, Lecce, Italy; ${ }^{2}$ Department of Medicine, Surgery and Neuroscience. University of Siena, Siena, Italy

Background: Many Rheumatic disease such as rheumatoid arthritis, juvenile idiopathic arthritis, psoriatic arthritis, ankylosing spondylitis, systemic lupus erythematosus, dermatomyositis/polymyositis and vasculitis are characterised by osteoporosis and fragility fractures. Inflammatory cytokines, steroid treatment, immobilization and reduced physical activity due to joint pain and muscle weakness are considered the major risk factors for the development of low bone mineral density in these diseases. Many evidences have highlighted the role of pro-inflammatory cytokines (TNF-a, IL-1, IL-6, IL-7, IL17) in bone homeostasis regulation.Chronic inflammation is often characterized by an imbalance between bone formation and resorption, with a clear prevalence of osteoclastogenesis which is a strong determinant in rheumatic diseases bone loss.

Objectives: The aim of this study is to evaluate the REMS (Radiofrequency Echographic Multi-Spectrometry) technology in rheumatologic patients, compared to DEXA currently recognised as the gold standard for the evaluation of bone mineral density.

Methods: Twenty female patients (mean age $60.6 \pm 14.41$ years) with different rheumatologic diseases were considered. Each patient underwent a lumbar spine and hip examination performed by DEXA and REMS technology. In particular, after a quality control to assess that both the exams were performed correctly, 18 lumbar and 20 femoral exams (DEXA vs REMS) were compared.

Results: As Expected the exams performed show a good diagnostic match ( $>60 \%$ LS and $>85 \%$ FEMORE). The tests that didn't show diagnostic concordance were those affected by arthrosis processes (greater on the Spine). The REMS T-score values were lower than those obtained with the DXA method. Conclusion: These results show how REMS technology can discriminate patients with osteoporosis as much as DEXA technology. The REMS technology can be a diagnostic option especially in patients with rheumatologic diseases that cause alterations in the spine reducing the diagnostic sensitivity of DXA technology.

Disclosure of Interests: None declared

DOI: 10.1136/annrheumdis-2020-eular.6500

\section{THU0637-HPR THE ASSOCIATION BETWEEN THE RECALL PERIOD AND THE AMOUNT OF INFORMATION ABOUT REPORTED ADVERSE DRUG REACTIONS BY PATIENTS USING BIOLOGICALS}

A. Laurijssen ${ }^{1}$, J. Van Lintt $^{2}$, B. Van den Bemt ${ }^{1,3}$, L. Beijer ${ }^{1,3}$, N. Jessurun ${ }^{2}$ ${ }^{1}$ Radboud University, Nijmegen, Netherlands; ${ }^{2}$ Pharmacovigilance centre Lareb, 's Hertogenbosch, Netherlands; ${ }^{3}$ Sint Maartenskliniek, Nijmegen, Netherlands

Background: In order to monitor the safety of medicines pharmacovigilance, it is important that patients report their adverse drug reactions (ADRs). Theoretically, the quality of the reported information might be affected by the elapsed time between the onset of the ADR and the moment of reporting. Real-life evidence demonstrating a negative relationship between this recall period and the quality of reported ADRs is however lacking.

Objectives: To assess the effect of recall period on the amount of information that patients report about their ADR (information density) in patients using a biologic for an immune-mediated inflammatory disease (IMID).

Methods: The Dutch Biologic Monitor is a multi-center cohort ADR monitoring system collecting data on reported ADRs by patients using a biologic for an IMID. Per patient, every first unique reported ADR between 1 February 2017 and 1 September 2019 was eligible. ADR reports were selected by stratified random sampling based on length of recall period and biologic. The recall period was defined by the number of days between the onset and reporting date of the ADR. The amount of information in an ADR report (information density) was determined based on eleven domains: specification, location, frequency, time-toonset, course, causality, cause or consequence, health care professional (HCP) visits, HCP action, patient action and ADR burden. Information density was calculated by the number of reported domains divided by the number of domains deemed relevant in the ADR report. The association between the information density of the ADR reports and different recall periods was compared using a one-way ANOVA test. One-way ANOVA and independent t-tests were used to assess the impact of gender, age, type biologic and burden of the ADR on the information density of the reported ADRs

Results: Out of 1109 reported ADRs by 531 IMID patients, we included 402 ADR reports of 294 patients (55\%) (see table 1 ). Included reports were equally divided over seven different recall periods: 0-1, 1-2, 2-4, 4-8, 8-12, 12-26 and 26-52 weeks. Results have shown no association between the information density in patient-reported ADRs and the length of recall period $(p=0.805)$ (figure 1). However, the proportion of reported information about HCP visits for the ADR increased with increasing recall period: 0 -1 week (14\%), 1-2 weeks $(24 \%), 2-4$ weeks (34\%), 4-8 weeks (40\%), 8-12 weeks (48\%), 12-26 weeks $(50 \%)$ and 26-52 weeks (46\%).

Table 1. Characteristics of included patients with adverse drug reactions

\begin{tabular}{lc}
\hline Characteristics (N=294) & $\mathbf{N}(\%)$ \\
\hline Gender (female) & $202(69 \%)$ \\
Age (years) (mean \pm SD) & $53 \pm 13$ \\
Smoking & $59(20 \%)$ \\
BMI (kg/m ${ }^{2}$ ) (mean \pm SD) & $25.7 \pm 5.3$ \\
Reported ARDs (mean \pm SD) & $1.4 \pm 0.8$ \\
Indication & \\
Rheumatoid arthritis & $129(44 \%)$ \\
Psoriatic arthritis & $51(17 \%)$ \\
Axial spondyloarthritis & $43(15 \%)$ \\
Crohn's disease & $42(14 \%)$ \\
Other indications & $29(10 \%)$ \\
Biologic & \\
Adalimumab & $97(33 \%)$ \\
Etanercept & $72(24 \%)$ \\
Infliximab & $27(9 \%)$ \\
Tocilizumab & $16(5 \%)$ \\
Secukinumab & $15(5 \%)$ \\
Rituximab & $14(5 \%)$ \\
Other biologics & $53(18 \%)$ \\
\hline
\end{tabular}

Female patients reported more information about their ADR $(p=0.002)$, whereas the patient's age was not associated with information density $(p=0.221)$. Etanercept $(E T A)$ users report significantly more information than adalimumab (ADA) users $(p=0.019)$. The number of patients using other biologics was too low for further analysis. A higher ADR burden tended $(p=0.120)$ to result in more reported ADR information (figure 2).

Conclusion: The length of recall period did not affect the amount of information that patients report about their $\operatorname{ADR}(\mathrm{s})$. The recall period was longer for patients reporting information about their HCP visit. Furthermore, female patients tend to report more information about their ADR than male patients and ETA-users tend to report more than ADA-users.

Figure 1: No effect of length of recall period on information density in patient-reported ADRs

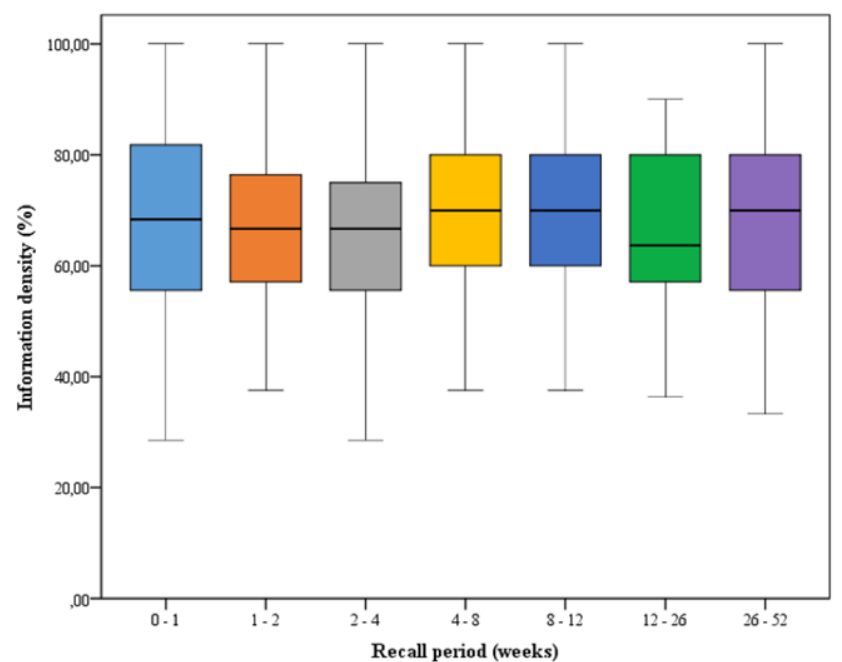

